\title{
AC 2010-1444: EXPERIMENTAL TESTING TO ENHANCE THE DESIGN OF DAYLIGHTING SYSTEMS. A CASE STUDY ON THE IMPLEMENTATION OF LABORATORY-ORIENTED DESIGN IN UNDERGRADUATE EDUCATION
}

Khaled Mansy, Oklahoma State University 


\title{
Experimental Testing to Enhance the Design of Daylighting Systems. \\ A Case Study on the Implementation of Laboratory-Oriented Design in Undergraduate Education
}

\begin{abstract}
This paper addresses a timely topic, which is designing and enhancing the performance of daylighting systems in buildings early in the design phase. Currently in academia, the design of daylighting systems lacks the emphasis, rigor, and time normally given to electric lighting systems. However, only in a few higher education institutions, more attention is being gradually given to the study of daylighting systems. Undoubtedly, the substantial environmental and economic benefits of daylighting make its study a must to include in both architecture and architectural engineering curricula. In this paper, the author shares his experience teaching undergraduate students the principles of the design of daylighting systems; implementing laboratory-oriented studies as the educational tool. The paper starts with a brief survey of the existing design-assisting tools, discusses three advanced design methodologies, which are computer programs, outdoor model testing, and indoor model testing, and then concludes with a discussion of the implementation of laboratory-oriented design in undergraduate education.
\end{abstract}

\section{Introduction}

Design of daylighting systems in buildings is an energy saving strategy. When commercial buildings rely more on natural light instead of electric light, they do not only save light energy, but also reduce the cost of air conditioning. That is mainly why daylight always occupies a high priority on the list of energy conservation measures (ECMs) for commercial buildings. For the same reason, integration of daylighting systems earns credit points for the LEED (Leadership in Energy and Environmental Design) rating system ${ }^{1}$ and other similar rating systems of green buildings. In fact, if daylighting systems are designed to completely replace the use of electric lighting systems in commercial buildings, they can yield significant energy savings that may reach up to $31.6 \%$ of site electricity consumption, which amounts to $24.8 \%$ of the total primary energy consumed by the commercial buildings sector in the US (2006) ${ }^{2}$.

Awareness of the importance of integration of daylighting systems in buildings is gradually and consistently growing in both academia and the industry. However, in a typical case in academia, the design of daylighting systems follows some common general rules of thumb and not any rigorous scientifically-sound design methodology. The main purpose of this paper is to highlight the importance of introducing rigorous experimental testing of scale models as a design-assisting tool to enhance the design of daylighting systems in buildings, while still in the early design phase. According to the author's experience, laboratory-testing proved to be both an effective and a transparent educational tool.

\section{Simplified Design Methods}

The rules of thumb currently used to integrate daylighting into architectural design can only offer general speculations such as: (1) within a depth of 15 feet from the window wall system daylight is sufficient, and up to 30 feet from the window wall system daylight provides $50 \%$ of required 
illumination levels, (2) within a depth that is equal to $250 \%$ of the difference in height between the workplane and the top of the window, daylight is sufficient ${ }^{3}$, (3) the average daylight factor in the space is equal to one tenth of the glass ratio of the exterior wall. These rules of thumb do not take into account influential design variables such as, size, design, and placement of windows; building location and orientation; hour in the day; target illumination level; and the overlap with daily and annual occupancy schedules ${ }^{4}$. Although the very simple feedback from these well-known rules of thumb may be useful, it does not help provide any further feedback on how to further develop and enhance the design of daylighting systems.

On the other hand, simplified mathematical and graphical design methods offer a better feedback than the rules of thumb. However they are based on pre-calculated databases, which are previously obtained from testing a set of physical models. Configuration of those models may not apply to other cases. Furthermore (for example), the simplified lumen method ignores the impact of the design and placement of windows. Similarly, the protractor method, first developed for the overcast conditions in the UK, does not give accurate results because it assumes a uniformly distributed sky luminance, which is now an invalid assumption. Using the protractor method is a very time consuming process ${ }^{5}$. The high level of uncertainty, associated with the use of simplified mathematical and graphical methods, results in their limited use, if they are used at all in academia or in professional practice.

In conclusion, the use of rules of thumb may be only useful very early in the design process during the preliminary design phase and cannot help enhance the design of daylighting systems later on in the process during the design development phase. The afore-mentioned simplified methods are limited in their use and are grossly outdated ${ }^{6}$.

\section{Advanced Design Methods}

Since simplified design methods are limited in the help they may offer, advanced design methods should be utilized in order to enhance the design of daylighting systems. These advanced design methods are identified in this paper as: (1) computer programs, (2) outdoor model testing, and (3) laboratory model testing. Each method is discussed below. In general, the comments address the use of each method as a teaching tool for undergraduate students.

\subsection{Computer Programs}

Recently, many computer programs have been developed to simulate the behavior of daylight inside buildings. The majority of these computer programs utilize the radiosity or ray-tracing technique to track the light emitted from the sun and the sky through windows and as reflected off interior surfaces, ultimately reaching the workplane and other interior surfaces inside the space. Some of these programs, such as Radiance and AGi32, can predict illuminance due to daylight in complex-shaped spaces to an acceptable level of accuracy. However, other programs have limitations on the geometry of spaces they may analyze, and some may not be able to simulate certain standard sky conditions.

Although accurate results can be obtained through the use of computer programs, the educational disadvantage is that: when beginning students use them, they tend to focus their attention on the end result of the simulation regardless of the process or the basic science behind how the system 
works. For them, computer programs are perceived as a trustworthy black box tool that is not transparent enough or does not encourage spending enough time to engage in the process.

Indeed, for experienced designers, computer programs are perceived as user-friendly and a timesaving design-assisting tool. On the contrary, for beginning students and designers, what helps their educational development is the use of a more transparent design-assisting tool, before they are ready to switch to computer programs.

Here, it is also worth-mentioning that when computer programs are utilized to perform computational research work, it is always recommended to validate the computational research results with experimental research. Indeed, this fact further highlights the importance of laboratory-oriented studies.

\subsection{Outdoor Model Testing}

In case experimental testing of daylighting systems is adopted as the design-assisting tool, the two possible choices are outdoor testing or indoor testing. In outdoor testing of daylighting models, students can attach the physical scale model to a tilting table, in a relatively open area in the outdoor (Figure 1a). In order to test the model under clear sky conditions (no cloud cover), they rotate and tilt the model until its relative horizontal and vertical solar angles (Azimuth and Altitude) match the Azimuth and Altitude of the sun at the location, day, and hour being tested, which can be done using the sundial for the appropriate latitude as shown in Figure 1b. Then, the students take illuminance readings inside and outside the model in order to obtain the Daylight Factor (DF). In case of testing the model under overcast sky conditions, illuminance readings can be taken when the table is flat horizontal in a relatively open area.
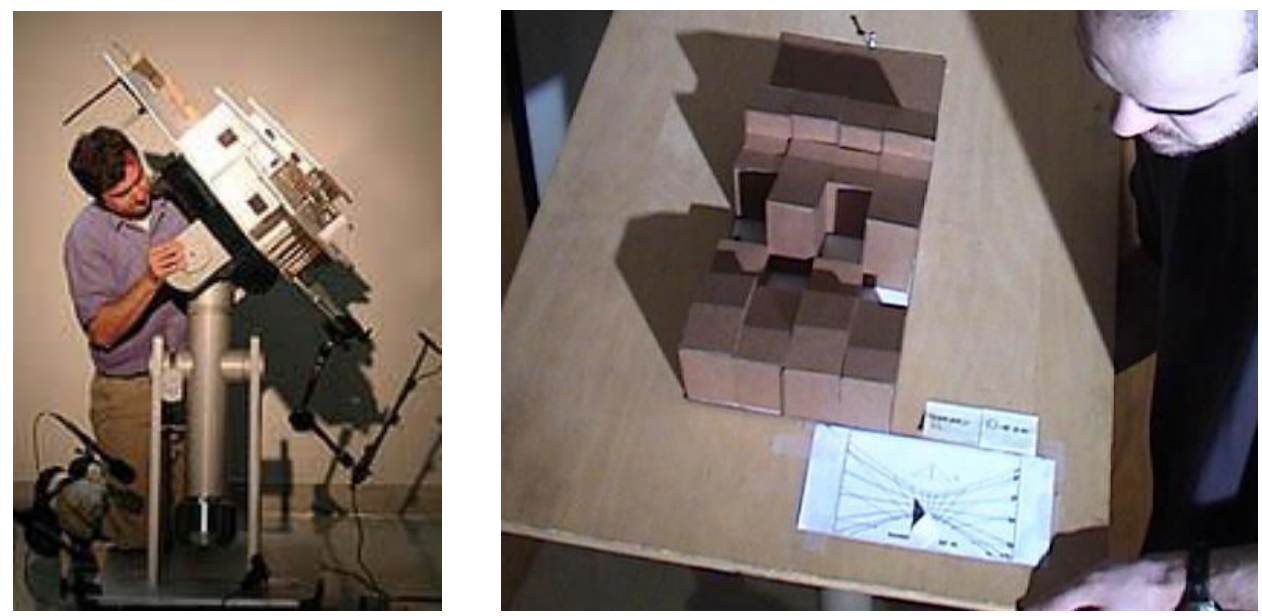

Figure 1a: Testing Daylight Models using the tilting table

Figure 1b: Adjusting the Azimuth and Altitude for the location, day, and hour

This technique of testing daylight scale (physical) models in the outdoor is often implemented by professional designers. However, it inherits the following disadvantages:

1. Although the model is tested under real outdoor sky conditions, these conditions are unlikely to match any of the CIE (Commission Internationale De L'Eclairage) standard sky conditions. Each standard CIE sky condition has a specific luminance distribution on 
the sky dome that is based on statistical analysis of extensive field measurements. That is why a real outdoor sky condition should not be considered a design condition.

2. Because outdoor sky conditions are constantly changing, it is almost impossible to retest an updated (improved) design of the same model under the same sky condition. Indeed, this may undermine the validity of any comparative analysis.

3. For the same reason in (2), taking several readings inside the model has to be done quickly enough before any significant changes of sky luminance or sky luminance distribution may occur.

4. The continuously changing brightness of the outdoor sky makes it extremely important to take readings inside and outside the model at the same moment (in order to calculate the Daylight Factor). If this fact is overlooked, it skews the results of the analysis.

5. In case of testing models under the clear sky and adjusting the tilting table to match a certain solar angle, the model becomes exposed to an incomplete hemispherical sky with exterior obstructions that do not necessarily represent the site simulated.

\subsection{Laboratory Model Testing}

Testing daylight physical models in a laboratory setting overcomes the above-mentioned disadvantages of outdoor testing since the indoor laboratory environment (in a windowless room) ensures full control over the luminous conditions inside the laboratory. For that purpose, the artificial sky dome (Figure 2) can be used to simulate specific sky conditions. As shown in Figure 2, the artificial sky dome consists of a translucent dome. The luminance distribution on its hemispherical surface is controlled by 341 light bulbs mounted on a larger concentric openframe dome. An electronic controller controls the brightness of every light bulb according to an algorithm that can create the luminance distribution of any standard CIE sky condition. When students use this laboratory (the dome), they place the scale model at the center point of the dome and adjust its orientation to match the orientation of the space tested. Then, they insert light sensors inside and outside of the model, turn the dome on, and take readings off a light meter that is connected to all of the light sensors.

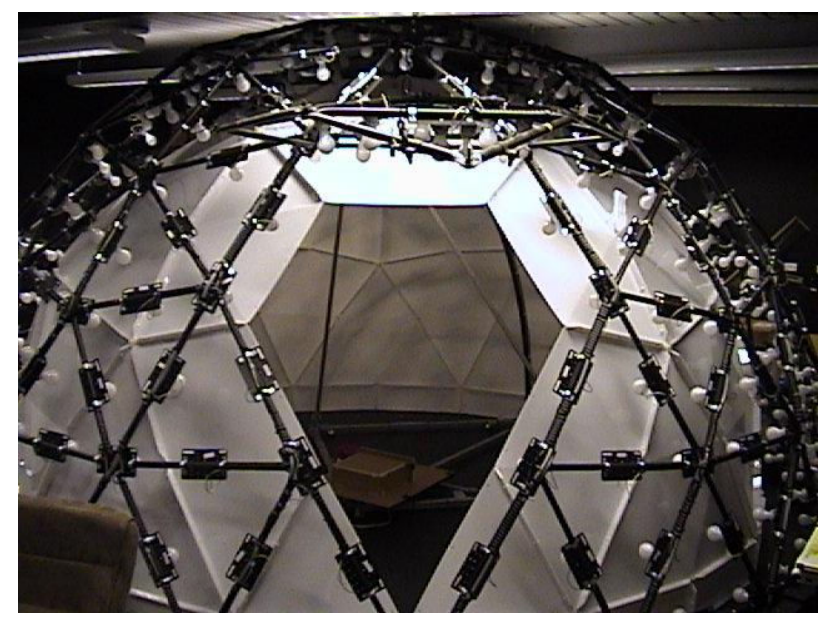

Figure 2: The Artificial Sky Dome Laboratory

Laboratory testing of daylight models proved to be reliable, accurate, and an engaging educational tool. Its advantages can be summarized as follows: 
1. Placing the artificial sky dome in a windowless laboratory space isolates it from the everchanging sky conditions in the outdoor.

2. Creating the sky luminance distribution using an electronic controller allows the repeated simulation of identical sky conditions necessary for performing comparative studies.

3. For the same reason in (2), it is possible to create any of the CIE standard sky conditions, according to the algorithms developed by CIE for that purpose.

4. It is possible to create any outside sky obstructions to represent real conditions on the site under consideration.

5. Students can test their models any time in the day (day and night), and regardless of any outdoor weather conditions. This makes scheduling of testing time flexible and better coordinated with students' schedules.

\section{Educational Objectives}

This paper reports on the author's experience in teaching the design of daylighting systems to undergraduate students. Rigorous design of daylighting systems was recently added as a required assignment in a capstone design studio that is required for architectural engineering students in one of the professionally-accredited schools with integrated programs in architecture and architectural engineering (see Figure 3). The same studio is also required for the architecture students.

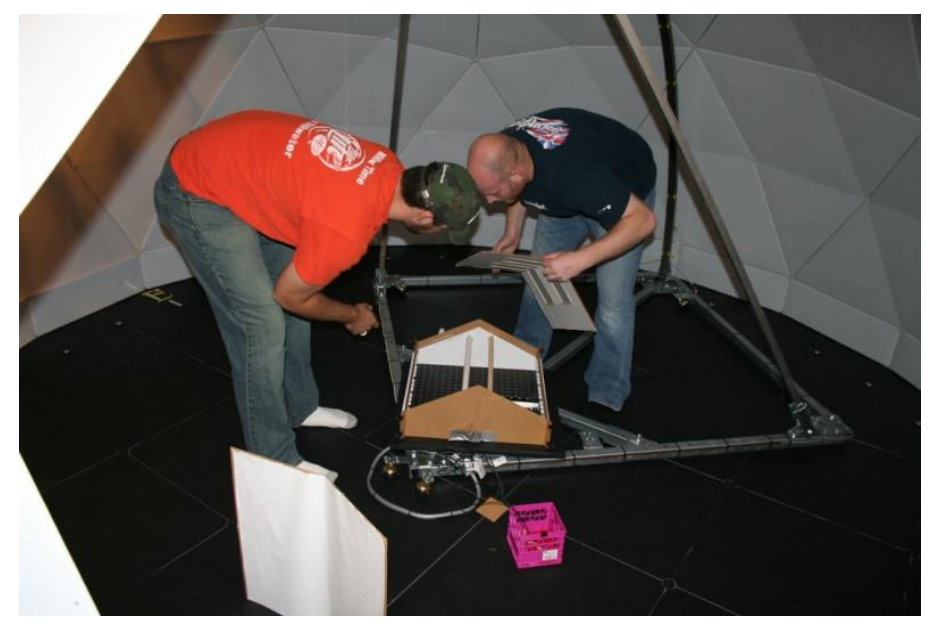

Figure 3: Students Testing a Model inside the Artificial Sky Dome

Using the artificial sky dome laboratory for experimental testing of the performance of daylighting systems in buildings achieved the educational goals set for this educational experience. Experimental laboratory testing provided the students with an interactive learning experience and an immersive engagement that helped them to understand the underlying scientific principles of the design of daylighting systems. Educational objectives of this laboratory-oriented study can be summarized as follows:

1. Awareness of the CIE standard sky conditions, and the ability to create the appropriate design condition for the location under consideration.

2. Understanding the effect of sky luminance distribution in association with window placement, space orientation, and sun position. This three dimensional relationship can be easily observed while testing the model under the sky dome. 
3. The ability to perform comparative analysis of alternative designs of daylighting systems in order to improve system's performance.

4. The ability to perform parametric analysis and experimental research.

\section{Logistics of Laboratory Testing}

Since a fundamental purpose of this paper is to share experience with peers, it is beneficial to mention the lessons learned through working with the students on their experimental design in the daylighting laboratory (the artificial sky dome). Undoubtedly, the biggest logistic advantage is the flexibility in scheduling lab time, which is almost impossible in case of outdoor testing. Logistic challenges can be summarized as below:

1. Scale of the physical models: in a typical space, interior illuminance readings should be taken at the height of the workplane, which is 30 inches above the floor. In this case, the scale of the model should be $1 / 2$ inch = 1'-00', and not any smaller, since the height of the LI-COR light sensor (including the base) is about $1 \frac{1 / 4}{4}$ inch.

2. In case the target illumination level (for the design) is at a higher level that the typical 30 inches, light sensors can be raised, or the scale of the model can be adjusted. However, in case the target illumination level is at the floor level, the light sensors should be embedded in the floor, which results in extra work for the students!

3. Testing scale models (indoor or outdoor) works very well for individual spaces. Testing the models indoor under the artificial sky dome works also for individual spaces even with multiple openings (windows and skylights) facing different orientations. However, when the space tested receives light from another adjacent space (open plan design), the model becomes bigger (or much bigger) in size and harder to build and to handle.

4. When students use a glass type that may modify the light distribution inside the space modeled, a sample of that glass type should be used. This is especially important with the use of Plexigls, or any other diffusing glass type.

\section{Conclusion}

Implementation of the laboratory-oriented testing as a design-assisting tool for daylighting systems (in buildings) is a successful example of evidence-based design. Testing physical models under the appropriate design sky condition in a fully-controlled laboratory setting assures the accuracy of the results; since this method takes into account all of the design variables that may influence the behavior of daylight inside enclosed spaces. Evidence-based design provides a valid scientific alternative to ungrounded speculations during the design phase, as well as the less accurate and/or inaccurate rules of thumb and outdated generalist design-assisting tools.

For comparison among the three reviewed design approaches, this laboratory-oriented educational experience shows that:

- Rules of thumb and simplified design methods cannot support enhancing the design of daylighting systems.

- Outdoor testing of daylight models eliminates the need for a daylighting laboratory, but may produce outcomes with questionable results, or makes it hard to perform comparative studies.

- Laboratory testing of daylighting models can provide accurate results, and its use is more reliable, flexible, and motivating. 
Building a daylighting laboratory (artificial sky dome) for the use of undergraduate and graduate students is possible. This artificial sky dome is designed and built using NSF funding. Total cost, including direct donations, is around $\$ 125,000$ for this 8 -foot radius dome. The lab room that houses the dome is about 24' $\mathrm{x} 30^{\prime}$ '. The dome does not require special maintenance; however, it was necessary to install an additional cooling equipment to carry the heating load from the 341 light bulbs in order to prevent any overheating of the dome and the lab room.

For more information on the design and capabilities of the artificial sky dome briefly described above, please refer to previous ASEE papers authored by the same author. Besides the information on the dome, previous papers also include a detailed description of the analytical method and calculations utilized for the evaluation of the performance of daylighting systems, i.e., the evaluation of energy savings due to daylighting.

\section{Bibliography}

1. USGBC (US Green Building Council), LEED-NC check list, http://www.usgbc.org, 2010.

2. DOE (US Department of Energy), 2008 buildings Energy Data Book, 2008.

3. Mansy, The Artificial Sky Laboratory at Oklahoma State University, proceedings of the 2004 American Society of Engineering Education Annual Conference \& Exposition, 2004.

4. Ibid.

5. Ibid.

6. Mansy, A user-friendly procedure to visualize the hourly quantitative performance of daylighting systems, Solar Energy 77, (2004), 373-380, 2004. 